\title{
The Ser/Thr Kinase MAP4K4 Controls Pro-Metastatic Cell Functions
}

\author{
Dimitra Tripolitsioti ${ }^{1}$, Michael A Grotzer ${ }^{1,2}$ and Martin Baumgartner ${ }^{1^{*}}$ \\ ${ }^{1}$ Department of Oncology, Children's Research Center, University Children's Hospital Zürich, August-Forel Strasse 1, CH-8008 Zürich, Switzerland \\ ${ }^{2}$ Department of Oncology, University Children's Hospital Zürich, Steinwiesstrasse 75, CH-8032 Zürich, Switzerland
}

*Corresponding author: Martin Baumgartner, Department of Oncology, University Children's Hospital Zürich, Children's Research Center, August-Forel Strasse 1, CH-8008 Zürich, Switzerland, Tel: +41 4463488 20; Fax: +41 4463488 59; E-mail: Martin.Baumgartner@kispi.uzh.ch

Received date: December 20, 2016; Accepted date: January 31, 2017; Published date: February 03, 2017

Copyright: @ 2017 Tripolitsioti D, et al. This is an open-access article distributed under the terms of the Creative Commons Attribution License, which permits unrestricted use, distribution, and reproduction in any medium, provided the original author and source are credited.

\begin{abstract}
The search for novel targeted therapies for major human conditions such as diabetes, cardiovascular diseases and cancer is a slow and costly process. Progress is often hampered by poor drug efficaciousness in the patients, low selectivity/specificity of the compounds and cellular evasion mechanism that are rather common in anti-cancer therapies. This is particularly true also for compounds inhibiting kinases, which in theory are optimal targets thanks to their druggable enzymatic activity. Novel targeting strategies are needed to reduce side effects and treatment failure caused by non-specific drug function and target resistance, respectively. An ideal compound will repress the relevant kinase effector function, while leaving kinase functions that are not disease-relevant unaltered. To achieve function-specific inhibition, the molecular mechanism of the drug target that governs the pathological process, must be identified.
\end{abstract}

The Ser/Thr kinase MAP4K4 is implicated in inflammatory and metabolic disorders and cancer progression. In this review, we describe the molecular effector functions of MAP4K4 that exert those activities and how they have been identified and characterized both in invertebrate organisms and mammals. We discuss how the modulation of the cellular cytoskeleton by MAP4K4 may be connected to pathological conditions such as aberrant angiogenesis and cancer metastasis, and we describe the molecular mechanisms that are so far known to be mechanistically involved in these processes.

Keywords: MAP4K4; Cytoskeleton; Cancer metastasis; Cancer progression

\section{Introduction}

MAP4K4 (Mitogen activated protein kinase kinase kinase kinase 4, also known as Hepatocyte progenitor kinase-like/germinal center kinase-like kinase (HGK) and Nck-interacting kinase (NIK)) is a serine/threonine protein kinase and member of the human STE20 family kinases; it was first identified as a kinase interactor of the adaptor protein Nck in mice [1] and short time later the human orthologue was cloned as well [2]. The MAP4K4 gene is located on chromosome 2 at position q11.2 and encodes from 33 exons a protein containing 1288 amino acids. Structurally, MAP4K4 contains an Nterminal kinase domain, a coiled-coil domain, a C-terminal hydrophobic leucine-rich citron homology domain $(\mathrm{CNH})$ and two putative caspase cleavage sites $[3,4]$. An interdomain connecting the kinase and the $\mathrm{CNH}$ domain is involved in protein-protein interactions but is structurally and functionally only poorly understood. Alternative splicing results in five isoforms that display differences in interdomain composition, while kinase and $\mathrm{CNH}$ domain are $100 \%$ homologous. MAP4K4 is well conserved and orthologues are found across species. Genetic interference and gene expression analyses have implicated MAP4K4 activity in a plethora of cellular functions relevant for physiological and pathophysiological processes, including organ development, systemic inflammation, metabolic disorders and cancer.

The astounding bandwidth of these functions, which are further detailed below, indicates the interaction of MAP4K4 with a range of structurally and functionally different effectors. Surprisingly however, only a few interactors have so far been described, highlighting the still incomplete understanding we currently have of how MAP4K4 exerts its functions. Most of the so far identified MAP4K4 interactors are functionally associated with the modulation of the actin and microtubule cytoskeletons. It is thus tempting to speculate that MAP4K4 modulates cell behavior via targeting the underlying cytoskeleton dynamics. In this review, we will particularly focus on the role of MAP4K4 in cancer and cancer progression and discuss how MAP4K4-controlled cytoskeleton dynamics could initiate and sustain the complex morphodynamic processes associated with cancer initiation and progression.

\section{Signaling Through MAP4K4}

Since its discovery in the late nineties, only relatively few details on the regulation of MAP4K4 activity and its substrates could be revealed. This is all the more surprising, considering the wealth of its implications in development, metabolic disorders and cancer. Convincing evidence in different mammalian and fly cell systems revealed MAP4K4 functions closely associated with the tumor necrosis factor alpha (TNFa)-induced c-jun N-terminal kinase (JNK) signaling pathway $[1,2,5,6]$. TNF $\alpha$-induced JNK activation by MAP4K4 involves signal transmission through the kinases TAK1, MKK4 and MKK7 [2]. Interestingly, MAP4K4 not only mediates TNF- $\alpha$ signaling but also promotes TNFa expression [2], while TNFa can also cause the specific increase in MAP4K4 expression via the transcription factors c-Jun and ATF2 $[7,8]$. On the one hand, this positive feed-back regulation of TNFa signaling through MAP4K4 is likely involved in physiological cell functions during development and for tissue homeostasis. On the 
other hand, aberrant TNFa signaling through MAP4K4 was also linked to pathophysiological processes using genetic ablation and pharmacological inhibition approaches.

These processes include insulin sensitivity [8], systemic inflammation [9], pathogen-dependent oncogenic progression [10,11] and vascular inflammation and atherosclerosis [12]. In addition to the TNFa-receptor, growth factors (GF) such as PDGF or EGF activate MAP4K4 through receptor tyrosine kinases (RTKs) [2] and trigger the phosphorylation and activation of the MAP4K4 substrates sodiumproton exchanger [1] (NHE1) [13], ezrin, radixin, moesin (ERM) family proteins [14] and actin-related protein [2] (Arp2) [15]. Genetic studies in $C$. elegans identified an interaction between the worm ortholog of MAP4K4-MIG-15-and integrin/PAT3 [16].

Whether integrin receptor engagement could activate MAP4K4 signaling analogous to the TNFa receptor or the RTKs described above is currently not known. However, compelling evidence indicates that MAP4K4 decreases surface availability of active integrins [17], for example by inactivating integrins via moesin phosphorylation, which concomitantly competes with talin for integrin binding [18], or by accelerating their endosomal trafficking [19].

Still in the context of integrin signaling, MAP4K4 was found to be phosphorylated by the focal adhesion kinase Pyk2 [20], which is regulated by intracellular calcium levels and activated by G-proteincoupled receptors [21].

Although not directly activated by transforming growth factor $B /$ bone morphogenic protein (TGFß/BMP), MAP4K4 as well as the closely-related TNIK and MINK promote inhibitory phosphorylation of Thr312 in the TGFß/BMP effector R-Smads1/2/3/5/8 [22].

Somewhat counterintuitively, MAP4K4 along with its relatives MAP4K1-6, were recently also found to phosphorylate and activate the kinase Large Tumor Suppressor 1/2 (LATS1/2) under conditions of serum starvation [23], which inactivates Yes-associated protein 1 (YAP1) in the evolutionary conserved Hippo pathway. Hippo pathway activation and phosphorylation-dependent cytosolic retention of the YAP transcriptional regulator is tumor suppressive, as it prevents proproliferative and anti-apoptotic YAP signaling [24].

Together, a still incomplete picture begun to emerge, where MAP4K4 activation in membrane proximal, upstream compartments occurs in response to TNFa receptor, RTKs, and-possibly-also Gprotein-coupled receptor ligation, to trigger JNK and Hippo pathway activation, sodium-proton exchange, actin polymerization and plasma membrane remodeling (Figure 1).

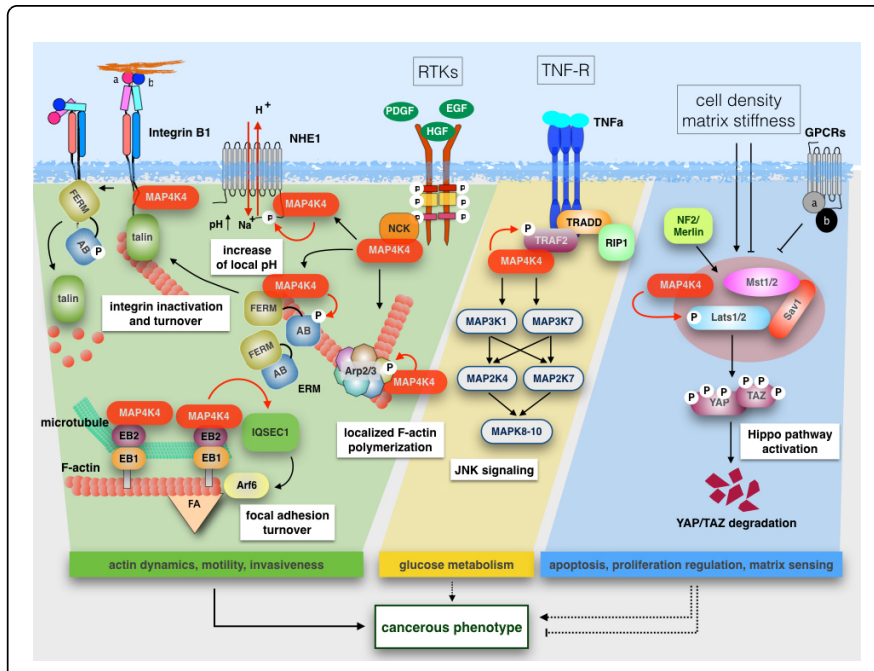

Figure 1: Overview of the major MAP4K4 signaling pathways.

\section{From Insulin Resistance to Cell Motility: The Panoply of MAP4K4 Control of Physiological Processes}

\section{Glucose up-take and insulin function}

The pathological failure of cells to respond to insulin with glucose up-take and metabolization is referred to as insulin resistance. It causes blood glucose levels to raise above the normal range, is associated with obesity, hypertension and cardiovascular diseases and eventually results in type 2 diabetes $[25,26]$. Causative mechanisms for insulin resistance include impaired insulin receptor (IR) signaling and the concomitantly deficient ability to restrict hepatic glucose output, as well as reduced uptake of glucose into muscle and adipose tissue through the glucose transporter GLUT4. An siRNA screen identified MAP4K4 as a negative regulator of GLUT4 control of glucose metabolism in adipocytes that impairs GLUT4 expression and function 8. Inflammation-associated $\mathrm{TNF} \alpha$ regulates insulin action negatively by repressing signaling downstream of the insulin receptor and by preventing GLUT4 translocation. Depletion of MAP4K4 ameliorates systemic inflammation in a murine model by reducing TNFa expression in macrophages [9]. This led to the hypothesis that MAP4K4 could also mediate the detrimental effects in glucose metabolizing cells exposed to systemic TNFa. Indeed, silencing MAP4K4 reverted TNFa-dependent IRS2 depletion and insulin repression in beta cells [27], and it restored peroxisome proliferatoractivated receptor gamma (PPARgamma) expression by de-repression of mTORC for a normalized glucose and lipid metabolism in adipocytes [28]. Consistently, microRNA 30d repression of MAP4K4 expression in pancreatic cells protected beta-cells against TNFainduced suppression of both insulin transcription and secretion, further confirming MAP4K4 targeting as a beneficial strategy for diabetes prevention [29].

MAP4K4 has also been suggested as a potential biomarker in nonobese type 2 diabetes, since decreased MAP4K4 expression ratesattributed to increased methylation of the MAP4K4 promoter-were observed in non-obese type 2 diabetes patients compared to healthy subjects [30]. Further in vivo studies demonstrated that MAP4K4 deletion led to decreased fasting blood glucose concentrations and 
enhanced insulin signaling in adipose tissue and liver [31], and that MAP4K4 mediates hyperinsulinemia in chronic obesity by promoting islet hypertrophy and insulin secretion from pancreatic cells [32]. MAP4K4 is also implicated in vascular inflammation and the formation of atherosclerotic plaques, where it enhances TNFa-induced vascular endothelial permeability, macrophage recruitment and adhesion [12].

These clear indications of the functional significance of MAP4K4 in inflammation-associated pathologies call for the use of therapeutics specifically targeting MAP4K4. However, the discovery of conditional MAP4K4 deficiency in T cells causing systemic inflammation and insulin resistance in mice sound a note of caution [33]. In T cells, MAP4K4 directly phosphorylates TNF receptor-associated factor 2 (TRAF2), leading to its degradation, repression of IL-6 production and the concomitant suppression of Th17 cell differentiation, which triggers an insulin resistant phenotype. Another study revealed that MAP4K4 expression in CD4+ T-cells restricts lung inflammation in response to the environmental pollutant ozone-oxidized black carbon $(\mathrm{oBC})$, which is characterized by increased numbers of macrophages, lymphocytes, neutrophils, cytokine secretion and bronchoalveolar lavage fluid accumulation (BALF) [34]. Finally, mice lacking endothelial MAP4K4 displayed lymphatic defects such as dilated lymphatic capillaries, insufficient lymphatic valves, and impaired lymphatic flow [35], suggesting that beneficial effects of MAP4K4 targeting in the atherosclerotic blood vasculature may have negative impact on lymphatic drainage. Thus, careful future studies will be required to assess the cell-type specific functions of MAP4K4 and their role in metabolic and inflammatory diseases.

\section{Development and Differentiation}

The Drosophila ortholog of MAP4K4 - misshapen (MSN) - was initially identified as shape control gene in the Drosophila eye [36]. Subsequent studies implicated it upstream of Drosophila JNK to control dorsal closure during late embryogenesis [5]. In mammals, MAP4K4 plays a critical role in stimulating migration of presomitic mesodermal cells away from the Primitive Streak (PS) and in promoting the differentiation of presomitic mesoderm into somites [37]. Its absence causes embryonic lethality between days 9.5 and 10.5. MAP4K4 is also involved in the control of epithelial to mesenchymal transition (EMT) during gastrulation, where it along with the p38interacting protein (p38IP) activates p38 to downregulated E-cadherin expression [38]. E-cadherin down-regulation contributes to the mesenchymal phenotype, which is prerequisite for cells to move away from the primitive streak. Interestingly, the above signaling cascade functions independently from the well characterized EMT-associated pathway that involves FGF and the Snail transcription factor, and suggests an alternative route requiring MAP4K4 activation of p38 [38].

TGFß/BMP signaling, which is involved in early development, axis formation and patterning [39], is negatively regulated by MAP4K4 and prevented from exerting its correct function during Drosophila imaginal disk formation [22]. Although speculative, MAP4K4 could thus be involved in spatio-temporal fine tuning of TGFß/BMP signaling during development. It is also conceivable, that alterations in this regulation in the context of aberrant MAP4K4 activation could result in developmental defects in the embryo and cancer initiation/ progression in the adult organism. In addition to its role in the developing organ, MAP4K4 also contributes to reshaping the adult organism, where it restricts differentiation of myoblasts into myotubes [40] or in enteroblasts, where it controls differentiation by phosphorylation of the Hippo pathway core component LATS1/2 and concomitant degradation of the transcriptional regulators YAP/TAZ [41].

\section{Axon Navigation and Neurite Outgrowth}

Axonal migration during development is a complex process that controls the correct orientation and connection of axons. MAP4K4 is involved in axonal navigation and neuronal pathfinding with the first evidence being identified in Drosophila with the identification of Msn/ MAP4K4 control of photoreceptor pathfinding [42]. Shortly after, the genetic interaction of MIG-15/MAP4K4 and the integrin Ina-1 was found to be required for proper axon navigation in C. elegans [16]. While MIG-15 appears to be dispensable for lamellipodia and filopodia dynamics during growth cone outgrowth and cell migration, it is required for proper directional control of neuronal outgrowth [43]. Comparative time lapse imaging of growth cone dynamics revealed that MIG-15 mechanistically interacts with the ezrin/radixin/moesin ortholog ERM-1 at the rear end of the growth cone, to restrict lateral protrusions and to maintain directional migration [44]. In mammalian cells, MAP4K4 was identified in a high-throughput screen for regulators of neurite outgrowth. In contrast to its neuro-promoting functions during development, MAP4K4 depletion in cerebellar granule neurons significantly increased the length of regenerating neurites, suggesting that MAP4K4 in mammals restricts neuronal outgrowth [45]. This finding was confirmed by an unrelated study using a militarinone-inspired 4-hydroxy-2-pyridone inhibitor targeting MAP4K4, which resulted in neuritogenesis [46]. MAP4K4 inhibition is also a reported target of kenpaullone, an inhibitor of Amyotrophic Lateral Sclerosis (ALS) [47]. Consistent with a potentially destructive function of MAP4K4 for neuronal integrity, kenpaullone improved the survival of human motor neurons derived either from mouse embryonic stem cells or from ALS-patient-induced pluripotent stem cells. Interestingly, kenpaullone efficiency was partially attributed to its inhibition of the MAP4K4-TAK1-MKK4-JNK-c-Jun cell death signaling cascade.

Together, these somewhat conflicting results from developing organisms and mature neurons corroborate the relevance of proper MAP4K4 function for neuronal development and integrity. However, future research will be necessary to reconcile the stabilizing functions of MAP4K4 during neuronal development with its destructive signaling in mature neurons. It will also be of particular interest to explore whether kenpaullone could be used to treat brain cancers with increased MAP4K4 expression such as glioblastoma [3,48] and medulloblastoma [49].

\section{MAP4K4 is Overexpressed in Solid Tumors}

A comparative analysis of healthy tissues with lines of the National Cancer Institute tumor panel reveled striking up-regulation of MAP4K4 in latter, with glioblastoma cancer cell lines displaying the highest upregulation in MAP4K4 expression levels [3]. Since then, a number of studies listed below have noted increased expression and/or altered function of MAP4K4 in tumors.

\section{Brain tumors}

Transcription analyses in glioblastoma multiforme cell lines and primary samples revealed that EGFRvIII, a constitutively activated EGFR mutant, leads to specific upregulation of a group of genes including MAP4K4 [48]. Also in a glioblastoma model, MAP4K4 was 
found to interact with the focal adhesion-related kinase Pyk2 and to cooperate with it for glioblastoma cell migration [20]. MAP4K4 expression is also markedly higher in pediatric Medulloblastoma, particularly in the $\mathrm{SHH}$ and Group 4 subgroups, compared to healthy cerebellum tissue, and promotes tumor cell migration and invasion downstream of the hepatocyte growth factor receptor c-Met [49].

\section{Pancreatic ductal adenocarcinoma}

MAP4K4 is overexpressed in $46 \%$ of stage II Pancreatic Ductal Adenocarcinoma (PDA) primary samples, with particular overexpression detected in the neoplastic epithelium compared to the PDA stroma [50]. Furthermore, multivariate statistical analyses in PDA samples revealed the association of MAP4K4 expression with poor overall and recurrence-free survival, recommending it as a prognostic marker for patients with stage II PDA [51].

\section{Colorectal cancer}

MAP4K4 and four additional genes (LYN, SDCBP, DKK1, and MID1) consist a five-gene signature in colorectal cancer highly correlated with poor overall survival of the patients. In addition, increased MAP4K4 mRNA expression per se closely correlates with metastasis, tumor invasion and decreased overall patient survival [52].

\section{Lung adenocarcinoma}

Adenocarcinoma is the most common histological subtype of lung cancer and accounts for almost half of all lung cancers. Elevated MAP4K4 expression is closely associated with lung adenocarcinoma progression and is an independent prognostic factor predicting poor overall survival [53].

\section{Hepatocellular carcinoma (HCC)}

MAP4K4 expression is increased in Hepatocellular carcinoma (HCC) specimens compared to adjacent non-tumor liver tissues, and its upregulation correlates positively with tumor size, tumor grade and intrahepatic metastasis. Consistently, high MAP4K4 expression is an independent predictor of poor prognosis in HCC patients [54].

\section{Gastric cancer}

Gastric cancer is the second most common cause of cancerassociated mortality worldwide. In more than $2 / 3$ of gastric cancer patient samples increased MAP4K4 expression levels were detected compared to adjacent non-tumorous regions [55].

\section{Ovarian cancer}

In contrast to all other solid tumors investigated, MAP4K4 expression levels correlate negatively with tumor load in a murine model of ovarian cancer. In this study, non-transformed ovarian epithelial cells were compared to their spontaneously transformed counterparts using genome-wide transcriptome analysis. Alterations in the gene expression profile were then correlated with the profiles of four different human ovarian cancer types [56].

\section{microRNA regulation of MAP4K4 expression in cancer}

microRNAs (miRNAs) are endogenous, small noncoding RNAs, with a length of approximately [19-24] nucleotides that negatively regulate gene expression by base pairing complementary sites on the 3'-untranslated region (3'-UTR) of target messenger RNAs [57]. Several MAP4K4-targeting miRNAs were found repressed in tumors, leading thus to increased levels of MAP4K4 expression. These include Let7a in Kaposi sarcoma, miR-194 in CRC [58] and HCC [59] and miR-622 in CRC [60].

\section{MAP4K4 Controls Pro-Metastatic Functions in Cancer Progression}

Increased expression levels of MAP4K4 at mRNA or protein levels have been correlated with tumor aggressiveness and reduced patient survival. But what are the molecular events controlled by MAP4K4 that trigger or maintain the transformed cellular phenotype and promote metastatic disease? Despite research efforts ongoing for close to 20 years, this question still remains incompletely answered. Improved gene interference strategies and the development of small compound inhibitors of MAP4K4 have simplified the identification of effector pathways and putative molecular interactors. Nevertheless, the effector molecules of MAP4K4 and how their interaction with altered MAP4K4 expression or function is coupled to the biological outcome, remains enigmatic in most instances. Key biological functions controlled by MAP4K4 are reviewed in the following section.

\section{Tumor cell growth}

Ras-transformation of NIH3T3 fibroblasts enables these cells to grow independent of adhesion to a rigid matrix (anchorage independent growth). Ablation of MAP4K4 function in such NIH3T3 fibroblasts by the overexpression of a kinase-inactive MAP4K4 mutant completely abrogated anchorage independence, suggesting that MAP4K4 contributes an essential function to anchorage-independent survival and growth [3]. Knock-down of MAP4K4 caused cell cycle arrest and apoptosis in HCCs [54] and in gastric cancer cells (GCCs) [55]. Cell cycle arrest and apoptosis after MAP4K4 knock-down was accompanied by the repression of JNK, NF kappa B, and toll-like receptor (TLR) signaling in HCC cells [54], and increased the Bax/ Bcl-2 ratio and decreased Notch signaling in GCCs [55]. Whether impaired HCC and GCC growth after MAP4K4 ablation is indeed the consequence of repressed JNK, NF-kappa B and TLR signaling, or rather mediated by an alternative route in these cancer cell lines is not clear. However, since MAP4K4 ablation does not cause growth arrest and apoptosis in all cancer cell lines, the susceptibility factor in the sensitive ones remains to be determined.

\section{Cell migration and invasion}

Early studies in Drosophila and C. elegans pointed towards cell shape and migration control as hallmark functions of MAP4K4 signaling [5,36,61]. Since disturbed morphodynamics and cell migration control are intimately associated with the cancerous phenotype, studies in cancer cell lines soon confirmed the promigratory function of MAP4K4 [3,62]. MAP4K4 implications in migration control in the context of cell transformation was also shown in macrophages infected with the protozoan parasite Theileria annulata, where parasite-induced TNFa secretion by the host cell activates MAP4K4 in an autocrine manner, to drive migration and invasion of the host cell [11]. In an inflammatory context as well, MAP4K4 promotes Kaposi Herpes virus-infected cell migration by controlling the expression of genes responsible for motility and invasiveness such as COX-2, MMP-7 and MMP-13. In HCC, MAP4K4 depletion markedly reduced cancer cell migration both in vitro and in 
vivo, reduced cell adhesion to extracellular matrix (ECM) proteins and impaired the expression of NF- $\kappa \mathrm{B}$ and the Matrix Metalloproteases 2 and 9 [54,63]. In the malignant pediatric brain tumor medulloblastoma, MAP4K4 is overexpressed and mediates migration and invasion downstream of HGF and EGF [49], whereas in adult glioblastoma cells, MAP4K4 was found to interact with Pyk2, a member of the focal adhesion kinase family, and to be required for migration and invasion [20]. Molecular mechanisms linking MAP4K4 activity to pro-metastatic functions Figure 2.

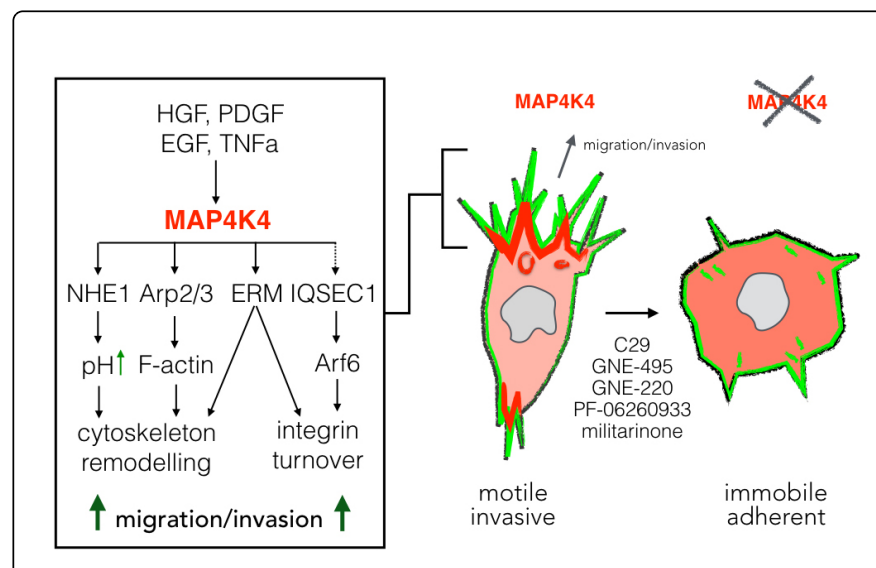

Figure 2: Upregulation of F-actin cytoskeleton turnover and membrane dynamics at the leading edge of cancer cells facilitate migration and tissue invasion. Small compound inhibitors targeting MAP4K4 block these pro-migratory functions and cells revert to a more stationary and less migratory phenotype.

Mechanistically, the identification of $C$. elegans integrin as an interaction partner of MAP4K4 during commissural axon navigation [16] hinted towards functional implication of MAP4K4 in cell adhesion and migration control. The ezrin, radixin, moesin ERM family were then identified as MAP4K4 substrates and interactors, which mediate lamellipodium extension in response to growth factor (GF) stimulation in breast adenocarcinoma cells [14] and in highly motile macrophages transformed by intracellular pathogen Theileria annulata [11]. Interestingly, in C. elegans, MIG-15 and the moesin orthologue ERM-1 function in the same pathway to direct commissural axon migration [44]. Mechanistically, MIG-15 and ERM-1 enable the stabilization of the rear end of the growth cone and the retraction of non-productive protrusions. Whether this structural stabilization of the growth cone by MIG-15 and ERM-1 in C. elegans is mechanistically related to lamellipodium stabilization in mammalian cells is not clear. Consistent with the notion that MAP4K4 controls cell adhesion are two recent studies describing accelerated turnover of integrin-based adhesions: On the one hand Yue et al. identified MAP4K4 as a focal adhesion (FA) disassembly factor acting in conjunction with microtubule ending binding protein 2 (EB2) and IQ motif and SEC7 domain-containing protein 1 (IQSEC1) in keratinocytes [64]. On the other hand, Vitorino et al. found that endothelial cell MAP4K4 phosphorylates moesin to displace the Factin binding protein talin from the intracellular tail of $B 1$ integrin [18]. Common to both studies is that MAP4K4 activity triggers the turnover of adhesion complexes. Indeed, depletion of MAP4K4 or its inactivation causes the cells to spread and adhere more tightly $[3,11,14]$. Acceleration of focal adhesion turnover may thus be one function of MAP4K4 to facilitate cell displacement, in particularly also inside 3D matrix, where focal adhesions, the adhesion associated signaling complexes and their coordinated turnover controls mesenchymal cancer cell migration [65].

Cytoskeleton dynamics at the cell front during migratoion are controlled among many other factors by local $\mathrm{pH}$ gradients. The sodium/proton $(\mathrm{Na}+/ \mathrm{H}+)$ exchanger 1 (NHE1) causes spatiallyrestricted $\mathrm{H}+$ efflux at the leading edge of cells [66]. In addition to its exchanger activity, NHE1 also acts as a membrane anchor for the cytoskeleton and an assembly point for signaling molecules [67-69]. MAP4K4 binds to and phosphorylates the C-terminus of NHE1 and causes its activation, which increases the intracellular $\mathrm{pH}$ in proximity of the plasma membrane [13]. NHE1 is involved in cell migration control [70-72] and associated with metastatic functions in cancer cells [73-75]. GF control of this central regulator of cortical cell functions through MAP4K4 may contribute to local remodeling of the cytoskeleton [76,77] and enable the formation of invasive membrane protrusions. Not surprisingly, targeting NHE1 has emerged as a potential strategy in tumor treatment [78].

Another possible function of MAP4K4 in cell migration and invasion control is its activity towards the actin-related protein 2 (Arp2) subunit of the Arp2/3 complex. Arp2 phosphorylation by MAP4K4 increased the actin nucleation activity of the Arp2/3 complex in vitro and in EGF-stimulated cells, demonstrating that MAP4K4 can couple growth factor (GF) signaling to F-actin polymerization [15]. MAP4K4 accumulates at the leading edge of cells migrating on/in either 2D or 3D matrix $[3,11,14,49,79]$. Thus, MAP4K4-driven F-actin polymerization at the leading edge combined with its activity towards focal adhesions make it a prime candidate for GF control of the membrane protrusions at the leading edge of cells that drive invasive cancer cell motility. Indeed, depletion of MAP4K4 in medulloblastoma cells infiltrating collagen I matrix caused a defect in protrusion formation and completely abrogated GF-induced cancer cell invasion [49].

\section{Therapeutics Targeting MAP4K4 Functions}

The broad implication of MAP4K4 in human disease conditions including metabolic and inflammatory disorders as well as cancer have incited efforts in the pharmaceutical industry to develop a specific small compound inhibitor. Genentech applied two separate fragmentbased lead discovery approaches, which identified pyridopyrimidine scaffold-based compound 29 (C29) [80] and C35 [40]. Starting from a micromolar pyrrolotriazine fragment hit, Genentech developed a series of MAP4K4 inhibitors with single digit nanomolar IC50s by utilizing crystal structures and molecular modeling. Calculated properties were used to guide the optimization of solubility and in vitro stability, yielding compounds with excellent biochemical potencies, ligand efficiencies and favorable in vivo bioavailability in mice. However, as with many small compound kinase inhibitors, moderate kinase selectivity was observed and the closely related kinase Misshapen-like kinase 1 (MINK1) and TRAF2- and Nck-interacting kinase (TNIK) were also effectively inhibited by both compounds. C35 was found to be even less specific as it also blocked activities of Cdk1, Flt3, KDR, KHS1, and Mink1 by more than 75\% [40]. Nevertheless, C29 was found to be a valuable tool in in vivo studies but its applicability suffered from the shortcoming that it was poorly tolerated when dosed repeatedly [80]. Its capability to permeate into the CSN was suspected to cause the poor tolerability. Medical chemistry efforts were therefore undertaken to reduce penetration of C29 into the CNS. The resulting GNE-495 reduced brain exposure while maintaining potent activity 
and good kinase selectivity, and recapitulated the phenotypes of MAP4K4 inducible knockout mice [81]. In a search for pharmacologically active compounds targeting pathological angiogenesis, the GNE-220-yet another MAP4K4 inhibitor from Genentech-was identified. This compound prevented MAP4K4dependent talin replacement by C-terminally phosphorylated moesin from the intracellular domain of integrin $\beta 1$, and reversed membrane retraction defects in endothelial cells associated with pathological angiogenesis [18]

A virtual screening approach using the crystal structure of the MAP4K4 kinase domain was employed by Pfizer, and the compound PF-6260933 with excellent selectivity was developed [82]. However, similar to the Genentech compounds, PF-6260933 also inactivated TNIK and MINK. PF-6260933 was used in a study investigating MAP4K4 function in vascular inflammation and atherosclerosis. It phenocopied the MAP4K4 knock-down and ameliorated plaque development in a murine model for atherosclerosis [12].

MAP4K4 is highly expressed in the brain tissue and its implications in neurodegenerative disorders have been addressed in the context of a search for natural products with neurotrophic and/or neuroprotective activities [46]. One such bioactive compound - militarinone-A - was identified as metabolite of a Cordycept fungus. Militarinone-A analogs were generated and as their protein targets were not known, they were analyzed for kinase selectivity. To evaluate the neurotrophic activity of those Militarinone-A analogs, they were subjected to phenotypic screening in the human neuroblastoma cell line SH-SY5Y. Compound 11e enhanced neurite outgrowth and inhibited MAP4K4 kinase activity in vitro, making it a suitable starting compound for future studies related to neurological disorders and - possibly - also MAP4K4-dependent brain tumors [46].

\section{Concluding Remarks}

The amazing diversity of MAP4K4 pathological function in different human conditions calls for a more general reflection on the biological processes it is involved with, and how they are associated with a specific disease condition. In this context, the regulation of the actin cytoskeleton is a good example, as it not only enables cellular morphodynamics but also spatially controlled signaling, surface receptor trafficking and turnover and endocytic up-take, to name only a few. MAP4K4 modulates the remodeling of the F-actin cytoskeleton in response to growth factors (GF) such as HGF, EGF and PDGF or the pro-inflammatory cytokine $\mathrm{TNF}$, both by directly controlling polymerization dynamics as well as signaling mediators associated with cytoskeleton control. The tripartite mechanism control of F-actin remodeling by MAP4K4 through $\mathrm{pH}$ regulation [13], actin polymerization and branching [15] and F-actin anchoring [14], strongly argues for MAP4K4 effectively translating growth factor (GF) signaling into cytoskeleton alterations affecting not only the overall morphology of the cell, but also how signals are transmitted inside the cell and how surface receptors are made available. Such alterations also support the disease conditions MAP4K4 was found associated with, in particular in pathological angiogenesis and cancer metastasis. On the one hand, it will thus be of importance to further dissect the downstream effector mechanisms of MAP4K4 specific to the condition, in order to more specifically target its function in this process. On the other hand, the signaling functions of MAP4K4 should not be observed isolated, but rather in the context of its broader impact on the cytoskeleton. Consequently, inflammatory signaling triggered by TNF $\alpha$ and mediated by MAP4K4, should thus also be investigated under the angle of the effects MAP4K4 may have on cytoskeleton modulation. An example for such context-dependent, global modulation of cell function was observed in a host-pathogen model, where parasite-dependent, TNFa-induced MAP4K4 signaling in the host cells bifurcates into JNK expression and activation on the one hand and ERM protein-associated control of lamellipodium dynamics and invasive motility on the other hand [11]. Through its activity at different levels of cell function, specific targeting of its contextdependent actions may thus emerge as a therapy specifically tailored to the disease-causing activity, and hence reduce non-desired side-effects of treatment.

\section{Acknowledgments}

This work was supported by grants of the Swiss National Science foundation (SNF_31004A-144090/1) to M.B. and of the Childhood Cancer Research Foundation Switzerland to M.A.G.

\section{References}

1. Su YC, Han J, Xu S, Cobb M, Skolnik EY (1997) NIK is a new Ste20related kinase that binds NCK and MEKK1 and activates the SAPK/JNK cascade via a conserved regulatory domain. EMBO J 16: 1279-1290.

2. Yao ZB (1999) A novel human STE20-related protein kinase, HGK, that specifically activates the c-Jun $\mathrm{N}$-terminal kinase signaling pathway. J Biol Chem 274: 2118-2125.

3. Wright JH (2003) The STE20 kinase HGK is broadly expressed in human tumor cells and can modulate cellular transformation, invasion, and adhesion. Mol Cell Biol 23: 2068-2082.

4. Delpire E (2009) The mammalian family of sterile 20p-like protein kinases. Pflugers Arch 458: 953-967.

5. Su YC, Treisman JE, Skolnik EY (1998) The Drosophila Ste20-related kinase misshapen is required for embryonic dorsal closure and acts through a JNK MAPK module on an evolutionarily conserved signaling pathway. Genes and development 12: 2371-2380.

6. Liu H, Su YC, Becker E, Treisman J, Skolnik EY (1999) A Drosophila TNF-receptor-associated factor (TRAF) binds the ste20 kinase Misshapen and activates Jun kinase. Curr Biol 9: 101-104.

7. Tesz GJ (2007) Tumor necrosis factor alpha (TNF alpha) stimulates Map4k4 expression through TNF alpha receptor 1 signaling to c-Jun and activating transcription factor 2. J Biol Chem 282: 19302-19312.

8. Tang XQ (2006) An RNA interference-based screen identifies MAP4K4/NIK as a negative regulator of PPAR gamma, adipogenesis, and insulin-responsive hexose transport. P Natl Acad Sci USA 103: 2087-2092.

9. Aouadi M (2009) Orally delivered siRNA targeting macrophage Map4k4 suppresses systemic inflammation. Nature 458: 1180-1184.

10. Haas DA (2013) The inflammatory kinase MAP4K4 promotes reactivation of Kaposi's sarcoma herpesvirus and enhances the invasiveness of infected endothelial cells. Plos Pathog 9: e1003737.

11. Ma M, Baumgartner M (2014) Intracellular Theileria annulata Promote Invasive Cell Motility through Kinase Regulation of the Host Actin Cytoskeleton. Plos Pathog 10.

12. Roth Flach RJ, Skoura A, Matevossian A, Danai LV, Zheng W, et al. (2015) Endothelial protein kinase MAP4K4 promotes vascular inflammation and atherosclerosis. Nat Commun 6: 8995.

13. Yan W, Nehrke K, Choi J, Barber DL (2001) The Nck-interacting kinase (NIK) phosphorylates the $\mathrm{Na}+\mathrm{-H}+$ exchanger NHE1 and regulates NHE1 activation by platelet-derived growth factor. J Biol Chem 276: 31349-31356.

14. Baumgartner M, Sillman AL, Blackwood EM, Srivastava J, Madson N, et al. (2006) The Nck-interacting kinase phosphorylates ERM proteins for formation of lamellipodium by growth factors. Proc Natl Acad Sci U S A 103: 13391-13396. 
15. LeClaire LL, Rana M, Baumgartner M, Barber DL (2015) The Nckinteracting kinase NIK increases Arp2/3 complex activity by phosphorylating the Arp2 subunit. J Cell Biol 208: 161-170.

16. Poinat P, De Arcangelis A, Sookhareea S, Zhu X, Hedgecock EM, et al. (2002) A conserved interaction between beta1 integrin/PAT-3 and Nckinteracting kinase/MIG-15 that mediates commissural axon navigation in C. elegans. Curr Biol 12: 622-631.

17. Lewellyn L, Cetera M, Horne-Badovinac S (2013) Misshapen decreases integrin levels to promote epithelial motility and planar polarity in Drosophila. J Cell Biol 200: 721-729.

18. Vitorino P, Yeung S, Crow A, Bakke J, Smyczek T, et al. (2015) MAP4K4 regulates integrin-FERM binding to control endothelial cell motility. Nature 519: 425-430.

19. Alanko J, Mai A, Jacquemet G, Schauer K (2015) Integrin endosomal signalling suppresses anoikis. Nat Cell Biol 17: 1412-1421.

20. Loftus JC, Yang Z, Kloss J, Dhruv H, Tran NL (2013) A Novel Interaction between Pyk2 and MAP4K4 Is Integrated with Glioma Cell Migration. J Signal Transduct 2013: 956580.

21. Dikic I, Tokiwa G, Lev S, Courtneidge SA, Schlessinger J (1996) A role for Pyk2 and Src in linking G-protein-coupled receptors with MAP kinase activation. Nature 383: 547-550.

22. Kaneko S, Chen X, Lu P, Yao X, Wright TG, et al. (2011) Smad inhibition by the Ste20 kinase Misshapen. Proc Natl Acad Sci U S A 108: 11127-11132.

23. Meng Z, Moroishi T, Mottier-Pavie V, Plouffe SW, Hansen CG, et al. (2015) MAP4K family kinases act in parallel to MST1/2 to activate LATS1/2 in the Hippo pathway. Nat Commun 6: 8357.

24. Huang JB, Wu S, Barrera J, Matthews K, Pan DJ (2005) The Hippo signaling pathway coordinately regulates cell proliferation and apoptosis by inactivating Yorkie, the Drosophila homolog of YAP. Cell 122: 421-434.

25. Biddinger SB, Kahn CR (2006) From mice to men: insights into the insulin resistance syndromes. Annu Rev Physiol 68: 123-158.

26. Kahn SE, Hull RL, Utzschneider KM (2006) Mechanisms linking obesity to insulin resistance and type 2 diabetes. Nature 444: 840-846.

27. Bouzakri K, Ribaux P, Halban PA (2009) Silencing Mitogen-activated Protein 4 Kinase 4 (MAP4K4) Protects Beta Cells from Tumor Necrosis Factor-alpha-induced Decrease of IRS-2 and Inhibition of Glucosestimulated Insulin Secretion. J Biol Chem 284: 27892-27898.

28. Guntur KVP, Guilherme A, Xue LT, Chawla A, Czech MP (2010) Map4k4 Negatively Regulates Peroxisome Proliferator-activated Receptor (PPAR) gamma Protein Translation by Suppressing the Mammalian Target of Rapamycin (mTOR) Signaling Pathway in Cultured Adipocytes. J Biol Chem 285: 6595-6603.

29. Zhao XM, Mohan R, Ozcan S, Tang XQ (2012) MicroRNA-30d Induces Insulin Transcription Factor MafA and Insulin Production by Targeting Mitogen-activated Protein 4 Kinase 4 (MAP4K4) in Pancreatic beta-Cells. J Biol Chem 287: 31155-31164.

30. Chuang HC, Wang JS, Lee IT, Sheu WH (2016) Epigenetic regulation of HGK/MAP4K4 in $\mathrm{T}$ cells of type 2 diabetes patients. Oncotarget 7: 10976-10989.

31. Danai LV (2015) Inducible Deletion of Protein Kinase Map4k4 in Obese Mice Improves Insulin Sensitivity in Liver and Adipose Tissues. Mol Cell Biol 35: 2356-2365.

32. Flach RJR (2016) Protein Kinase Mitogen-activated Protein Kinase Kinase Kinase Kinase 4 (MAP4K4) Promotes Obesity-induced Hyperinsulinemia. J Biol Chem 291: 16221-16230.

33. Chuang HC, Sheu WH, Lin YT, Tsai CY, Yang CY, et al. (2014) HGK/ MAP4K4 deficiency induces TRAF2 stabilization and Th17 differentiation leading to insulin resistance. Nat Commun 5: 4602.

34. Jin M, Chu H, Li Y, Tao X, Cheng Z, et al. (2016) MAP4K4 deficiency in $\mathrm{CD} 4(+) \mathrm{T}$ cells aggravates lung damage induced by ozone-oxidized black carbon particles. Environ Toxicol Pharmacol 46: 246-254.
35. Flach RJR (2016) Endothelial Mitogen-Activated Protein Kinase Kinase Kinase Kinase 4 Is Critical for Lymphatic Vascular Development and Function. Mol Cell Biol 36: 1740-1749.

36. Treisman JE, Ito N, Rubin GM (1997) misshapen encodes a protein kinase involved in cell shape control in Drosophila. Gene 186: 119-125.

37. Xue Y, Wang X, Li Z, Gotoh N, Chapman D, et al. (2001) Mesodermal patterning defect in mice lacking the Ste20 NCK interacting kinase (NIK). Development 128: 1559-1572.

38. Zohn IE, Li Y, Skolnik EY, Anderson KV, Han J, et al. (2006) p38 and a p38-interacting protein are critical for downregulation of E-cadherin during mouse gastrulation. Cell 125: 957-969.

39. Wu MY, Hill CS (2009) Tgf-beta superfamily signaling in embryonic development and homeostasis. Dev Cell 16: 329-343.

40. Wang M, Amano SU, Flach RJ, Chawla A, Aouadi M, et al. (2013) Identification of Map4k4 as a novel suppressor of skeletal muscle differentiation. Mol Cell Biol 33: 678-687.

41. Li Q, Li S, Mana-Capelli S, Roth Flach RJ, Danai LV, et al. (2014) The conserved misshapen-warts-Yorkie pathway acts in enteroblasts to regulate intestinal stem cells in Drosophila. Dev Cell 31: 291-304.

42. Su YC, Maurel-Zaffran C, Treisman JE, Skolnik EY (2000) The Ste20 kinase misshapen regulates both photoreceptor axon targeting and dorsal closure, acting downstream of distinct signals. Mol Cell Biol 20: 4736-4744.

43. Shakir MA, Gill JS, Lundquist EA (2006) Interactions of UNC-34 Enabled with Rac GTPases and the NIK kinase MIG-15 in Caenorhabditis elegans axon pathfinding and neuronal migration. Genetics 172: 893-913.

44. Teulière J, Gally C, Garriga G, Labouesse M, Georges-Labouesse E (2011) MIG-15 and ERM-1 promote growth cone directional migration in parallel to UNC-116 and WVE-1. Development 138: 4475-4485.

45. Loh SHY, Francescut L, Lingor P, Bahr M, Nicotera P (2008) Identification of new kinase clusters required for neurite outgrowth and retraction by a loss-of-function RNA interference screen. Cell Death Differ 15: 283-298.

46. Schröder P, Förster T, Kleine S (2015) Neuritogenic militarinone-inspired 4-hydroxypyridones target the stress pathway kinase MAP4K4. Angew Chem Int Ed Engl 54: 12398-12403.

47. Yang YM (2013) A Small Molecule Screen in Stem-Cell-Derived Motor Neurons Identifies a Kinase Inhibitor as a Candidate Therapeutic for ALS. Cell Stem Cell 12: 713-726.

48. Ramnarain DB (2006) Differential gene expression analysis reveals generation of an autocrine loop by a mutant epidermal growth factor receptor in glioma cells. Cancer Res 66: 867-874.

49. Santhana Kumar K, Tripolitsioti D, Ma M, Grählert J, Egli KB, et al (2015) The Ser/Thr kinase MAP4K4 drives c-Met-induced motility and invasiveness in a cell-based model of $\mathrm{SHH}$ medulloblastoma. Springerplus 4: 19 .

50. Badea L, Herlea V, Dima SO, Dumitrascu T, Popescu I (2008) Combined Gene Expression Analysis of Whole-Tissue and Microdissected Pancreatic Ductal Adenocarcinoma identifies Genes Specifically Overexpressed in Tumor Epithelia. Hepato-Gastroenterol 55: 2016-2027.

51. Liang JJ, Wang H, Rashid A, Tan TH, Hwang RF, et al. (2008) Expression of MAP4K4 is associated with worse prognosis in patients with stage II pancreatic ductal adenocarcinoma. Clin Cancer Res 14: 7043-7049.

52. Hao JM, Chen JZ, Sui HM, Si-Ma XQ, Li GQ, et al. (2010) A five-gene signature as a potential predictor of metastasis and survival in colorectal cancer. J Pathol 220: 475-489.

53. Qiu MH, Qian YM, Zhao XL, Wang SM, Feng XJ, et al. (2012) Expression and prognostic significance of MAP4K4 in lung adenocarcinoma. Pathol Res Pract 208: 541-548.

54. Liu AW, Cai J, Zhao XL, Jiang TH, He TF, et al. (2011) ShRNA-targeted MAP4K4 inhibits hepatocellular carcinoma growth. Clin Cancer Res 17: 710-720.

55. Liu YF (2016) Silencing of MAP4K4 by short hairpin RNA suppresses proliferation, induces G1 cell cycle arrest and induces apoptosis in gastric cancer cells. Mol Med Rep 13: 41-48. 
56. Urzua U (2006) Transcriptomic analysis of an in vitro murine model of ovarian carcinoma: Functional similarity to the human disease and identification of prospective tumoral markers and targets. J Cell Physiol 206: 594-602.

57. Bartel DP (2007) MicroRNAs: Genomics, biogenesis, mechanism, and function (Reprinted from Cell, vol 116, pg: 281-297). Cell 131: 11-29.

58. Wang B, Shen ZL, Gao ZD, Zhao G, Wang CY, et al. (2015) MiR-194, commonly repressed in colorectal cancer, suppresses tumor growth by regulating the MAP4K4/c-Jun/MDM2 signaling pathway. Cell Cycle 14 1046-1058.

59. Zhao Y, Li F, Zhang X, Liu A, Qi J, et al. (2015) MicroRNA-194 acts as a prognostic marker and inhibits proliferation in hepatocellular carcinoma by targeting MAP4K4. Int J Clin Exp Pathol 8: 12446-12454.

60. Ruan W, Pang P, Rao Y (1999) The SH2/SH3 adaptor protein dock interacts with the Ste20-like kinase misshapen in controlling growth cone motility. Neuron 24: 595-605.

61. Collins CS, Hong J, Sapinoso L, Zhou Y, Liu Z, et al. (2006) A small interfering RNA screen for modulators of tumor cell motility identifies MAP4K4 as a promigratory kinase. Proc Natl Acad Sci U S A 103: 3775-3780.

62. Han SX, Zhu Q, Ma JL, Zhao J, Huang C, et al. (2010) Lowered HGK expression inhibits cell invasion and adhesion in hepatocellular carcinoma cell line HepG2. World J Gastroenterol 16: 4541-4548.

63. Yue J, Xie M, Gou X, Lee P, Schneider MD, et al. (2014) Microtubules regulate focal adhesion dynamics through MAP4K4. Dev Cell 31: 572-585.

64. Wolf K (2013) Physical limits of cell migration: control by ECM space and nuclear deformation and tuning by proteolysis and traction force. J Cell Biol 201: 1069-1084.

65. Putney LK, Denker SP, Barber DL (2002) The changing face of the $\mathrm{Na}+\mathrm{H}$ + exchanger, NHE1: structure, regulation, and cellular actions. Annu Rev Pharmacol Toxicol 42: 527-552.

66. Denker S, Tominaga T, Barber DL (1998) NHE1 links actin cytoskeleton to the plasma membran by directly interacting with ERM proteins. Mol Biol Cell 9.

67. Denker SP, Huang DC, Orlowski J, Furthmayr H, Barber DL (2000) Direct binding of the $\mathrm{Na}-\mathrm{H}$ exchanger NHE1 to ERM proteins regulates the cortical cytoskeleton and cell shape independently of $\mathrm{H}(+)$ translocation. Mol Cell 6: 1425-1436.

68. Baumgartner M, Patel H, Barber DL (2004) Na(+)/H(+) exchanger NHE1 as plasma membrane scaffold in the assembly of signaling complexes. Am J Physiol Cell Physiol 287: C844-850.

69. Lagana A, Vadnais J, Le PU, Nguyen TN, Laprade R, et al. (2000) Regulation of the formation of tumor cell pseudopodia by the $\mathrm{Na}(+) /$ H(+) exchanger NHE1. J Cell Sci 113: 3649-3662.
70. Denker SP, Barber DL (2002) Cell migration requires both ion translocation and cytoskeletal anchoring by the Na-H exchanger NHE1. J Cell Biol 159: 1087-1096.

71. Frantz C, Karydis A, Nalbant P, Hahn KM, Barber DL (2007) Positive feedback between $\mathrm{Cdc} 42$ activity and $\mathrm{H}+$ efflux by the Na-H exchanger NHE1 for polarity of migrating cells. J Cell Biol 179: 403-410.

72. Cong D (2014) Upregulation of NHE1 protein expression enables glioblastoma cells to escape TMZ-mediated toxicity via increased $\mathrm{H}(+)$ extrusion, cell migration and survival. Carcinogenesis 35: 2014-2024.

73. Amith SR, Wilkinson JM, Fliegel L (2016) Na+/H+ exchanger NHE1 regulation modulates metastatic potential and epithelial-mesenchymal transition of triple-negative breast cancer cells. Oncotarget 7: 21091-21113.

74. Stüwe L, Müller M, Fabian A, Waning J, Mally S, et al. (2007) pH dependence of melanoma cell migration: protons extruded by NHE1 dominate protons of the bulk solution. J Physiol 585: 351-360.

75. Webb BA (2016) A Histidine Cluster in the Cytoplasmic Domain of the $\mathrm{Na}-\mathrm{H}$ Exchanger NHE1 Confers $\mathrm{pH}$-sensitive Phospholipid Binding and Regulates Transporter Activity. J Biol Chem 291: 24096-24104.

76. Meima ME, Webb BA, Witkowska HE, Barber DL (2009) The sodiumhydrogen exchanger NHE1 is an Akt substrate necessary for actin filament reorganization by growth factors. J Biol Chem 284: 26666-26675.

77. Loo SY, Chang MK, Chua CS, Kumar AP, Pervaiz S, et al. (2012) NHE-1: a promising target for novel anti-cancer therapeutics. Curr Pharm Des 18: $1372-1382$.

78. Ma M, Baumgartner M (2013) Filopodia and Membrane Blebs Drive Efficient Matrix Invasion of Macrophages Transformed by the Intracellular Parasite Theileria annulata. Plos One 8.

79. Crawford TD (2014) Discovery of Selective 4-Amino-pyridopyrimidine Inhibitors of MAP4K4 Using Fragment-Based Lead Identification and Optimization. J Med Chem 57: 3484-3493.

80. Ndubaku CO, Crawford TD, Chen H, Boggs JW, Drobnick J, et al. (2015) Structure-Based Design of GNE-495, a Potent and Selective MAP4K4 Inhibitor with Efficacy in Retinal Angiogenesis. ACS Med Chem Lett 6: 913-918.

81. Ammirati M, Bagley SW, Bhattacharya SK, Buckbinder L, Carlo AA, et al. (2015) Discovery of an in Vivo Tool to Establish Proof-of-Concept for MAP4K4-Based Antidiabetic Treatment. ACS Med Chem Lett 6: 1128-1133.

82. Firat-Karalar EN, Welch MD (2010) New mechanisms and functions of actin nucleation. Curr Opin Cell Biol 23: 4-13. 